\title{
Performance of a Multigap RPC prototype for the LHCb Muon System
}

\author{
P. Colrain ${ }^{\mathrm{a}}$, G. Corti $^{\mathrm{a}}$, L. de Paula ${ }^{\mathrm{b}, 1,2}$, M. Gandelman ${ }^{\mathrm{b}, 1,2}$, \\ J. Lamas-Valverde ${ }^{a}$, B. Marechal ${ }^{b, 1,2}$, D. Moraes ${ }^{b, 1,2}$, \\ E. Polycarpo ${ }^{\mathrm{b}, 1,2,3,4}$, B. Schmidt ${ }^{\mathrm{a}}$, T. Schneider ${ }^{\mathrm{a}}$, A. Wright ${ }^{\mathrm{a}}$ \\ ${ }^{\text {a } C E R N, C H ~-~} 1211$ Geneve 23, Switzerland \\ ${ }^{\mathrm{b}}$ LAPE/IF-UFRJ, CP 68528 Rio de Janeiro, Brazil
}

\begin{abstract}
Several technologies are under consideration for the muon system of the LHCb experiment. RPCs are one of the favourite candidates for the outer areas where the particle fluxes are expected to be at most some $\mathrm{kHz} / \mathrm{cm}^{2}$. This work describes the results obtained with a Multigap Resistive Plate Chamber prototype under various beam conditions at the CERN facilities.
\end{abstract}

\section{Introduction}

The LHCb experiment [1] is a single-arm spectrometer with forward angular coverage from $\sim 10 \mathrm{mrad}$ to $\sim 300 \mathrm{mrad}$. The LHCb Muon System is composed of five muon tracking stations interleaved in a longitudinally-segmented shield. It must provide muon identification and L0 muon trigger formation. For the latter, good time resolution for bunch-crossing identification and reasonable momentum resolution are required. Due to their excellent timing properties, RPCs are candidates for the outer part of the muon system (starting from a polar angle of $\sim 150 \mathrm{mrad}$ ). In this region, the rate capability required is about

1 Partially supported by European Community (contract CI1*_CT94-0118)

2 Partially supported by CNPq, Brazil

3 Partially supported by CAPES, Brazil

4 Corresponding author. Fax +41 22 7679425; e-mail Erica.Polycarpo@cern.ch 
1.6-4.0 kHz/ $\mathrm{cm}^{2}$, depending on the LHCb operating luminosity. This number has been conservatively estimated, in order to guarantee safe operation.

It has been shown by many authors that the rate capability of RPCs can be improved by operating them in avalanche or low gas amplification mode (e.g. [2]), using gas mixtures with a high content of freon gases, in particular tetrafluoroethane [3]. The addition of small amounts of $\mathrm{SF}_{6}$ has increased the range of voltages in which the chambers can operate without streamer formation [4]. Further improvements have been achieved by using lower resistivity bakelite plates $\left(\rho \sim 10^{10} \Omega \mathrm{cm}\right)[5]$. It has also been shown that dividing the gas volume into several gaps [6] results in higher rate capability. The advantages of using several gas gaps are that the time walk and resolution are limited by the small size of the subgaps and that the tolerance to irregularities is larger. With this motivation, two multigap RPC prototypes have been built, with active areas $24 \times 24 \mathrm{~cm}^{2}$ and $1.2 \times 2.2 \mathrm{~m}^{2}$.

This paper describes the tests of the small prototype. In the CERN PS $(\sim 3$ $\mathrm{GeV} / \mathrm{c} \pi)$ and SPS $(120 \mathrm{Gev} / \mathrm{c} \pi)$ beam lines, special emphasis has been given to the rate capability studies. The performance of the chamber has also been tested under continuous and uniform irradiation over the whole surface in the Gamma Irradiation Facility (GIF), where timing properties and cross-talk effects were also investigated.

\section{Characteristics of the MRPC prototype}

The chamber, whose cross section is shown in figure 1, is composed of four gas gaps, each of $0.66 \mathrm{~mm}$, preserved with nylon button spacers. The plates are $0.7 \mathrm{~mm}$ thick melamine-phenolic laminates, with bulk resistivity $5-6 \times$ $10^{11} \Omega \mathrm{cm}$. The HV plane consists of a graphite layer sprayed on the outer surface of the external resistive plate, while the readout plane is made of kapton foil with copper gold coated strips (1.3 cm wide and $24 \mathrm{~cm} \mathrm{long),} \mathrm{each}$ connected to an amplifier shaper discriminator chip ASD8-B [7]. The thresholds used were always $\sim 120 \mathrm{mV}$. The gas mixture was $96 \% \mathrm{C}_{2} \mathrm{H}_{2} \mathrm{~F}_{4} / 3 \%$ isoButane $/ 1 \% \mathrm{SF}_{6}$. In addition, $1 \%$ of water vapour was added to this gas mixture, in order to ensure stability of the electrical and mechanical properties of the plates. 


\section{Tests carried out in the PS and SPS beam lines}

\subsection{Experimental setup}

The trigger system was composed of two scintillators $\left(15 \times 15 \mathrm{~cm}^{2}\right.$ and $20 \times 20$

$\left.\mathrm{cm}^{2}\right)$ centered on the beam line. A hodoscope composed of 8 horizontal $\left(H c h_{1-8}\right)$ and 8 vertical $\left(V c h_{1-8}\right)$ scintillator strips $(0.8 \mathrm{~cm}$ wide and $8.0 \mathrm{~cm}$ long) with 1 $\mathrm{cm}$ pitch was placed between the large scintillators and used in the offline analysis. The MRPC prototype was placed immediately behind the hodoscope. A fiducial region of three chamber strips was used to measure the efficiency for minimum ionizing particles which crossed the intersection $\left(\mathrm{Hch}_{5}, \mathrm{Vch}_{5}\right)$ of the hodoscope, corresponding to the peak of the two-dimensional beam profile in figure 2. The signal from the strips was discriminated before feeding it to a 16-hit TDC (LeCroy 1176), operating in common-stop mode. A 15 ns time window was used to calculate the efficiency.

\subsection{Rate capability studies at the PS and SPS}

Figure 3 shows the efficiency measured at the CERN PS as a function of the applied high voltage for different rates measured in the $\left(H c h_{5}, V c h_{5}\right)$ cell of the hodoscope. The efficiency is integrated over the spill duration of $\sim 400 \mathrm{~ms}$. The knee of the plateau is shifted towards higher voltages when the rate increases, due to the increasing voltage drop across the resistive plates, which reduces the effective field in the gas volume.

In order to determine the effect of the irradiation time on the performance of the chamber, a further test was done at the CERN SPS, which has a spill duration of $\sim 2500 \mathrm{~ms}$. The efficiency was measured for several time intervals. The result is shown in figure 4, together with the rate as measured in the hodoscope peak. The drop in efficiency is on one hand due to the variation of the rate within the burst; on the other hand it is possible to see that for similar rates a significant efficiency drop with time occurs (e.g., for a rate of approximately $7.5 \mathrm{kHz} / \mathrm{cm}^{2}$, the efficiency drops from $90 \%$ to $70 \%$ ). Therefore we conclude that the rate capability as measured at the PS is not valid for high rates. A precise quantitative measurement of the efficiency drop would require more careful investigation. Since in GIF the chamber can be continuously irradiated at the same rate, it is the best available facility at CERN to test the performance of the present MRPC prototype with the given bulk resistivity. 


\section{Tests in the Gamma Irradiation Facility}

\subsection{Experimental Setup}

The experimental setup in GIF was similar to the one used in the PS and SPS. The chamber was placed at $1.08 \mathrm{~m}$ from the ${ }^{137} \mathrm{Cs}$ source, between the two large scintillators. In such a position, the whole surface of the chamber could be uniformly irradiated. A set of lead filters with different attenuation factors (AF) allowed the reduction of the gamma flux through the chamber. The muon beam coming from the SPS beam dump was used to monitor the performance of the prototype.

\subsection{Experimental Results}

\subsubsection{Measurement of rate and efficiency}

Figure 5 shows the efficiency for various voltages and several attenuation factors. The efficiency is corrected for the probability of finding a spurious hit in the same time window (typical values $\sim 0.1 \%$ ). The effective rate in the chamber was measured using the method described in [8] and a $\gamma$ sensitivity of approximately \#gaps/800 = 1/200 has been observed, which is in good agreement with other measurements [9]. The chamber reaches $97 \%$ efficiency at $18 \mathrm{kV}$ for a rate of approximately $1.9 \mathrm{kHz} / \mathrm{cm}^{2}$.

\subsubsection{Timing Properties}

Figure 6 shows the time walk in the chamber as a function of the measured effective rate. The mean time measured without source (lowest rate) was taken as reference. The time resolution, shown in the same plot, lies in the range 1.5-1.9 ns. For the two highest rates, the voltages are $18 \mathrm{kV}$, otherwise it is $17 \mathrm{kV}$, which is already well within the efficiency plateau. It is clear that the timing requirements of $\mathrm{LHCb}$ are fulfilled by this prototype.

\subsubsection{Cross Talk and Noise}

The cluster size is taken as a measurement of the cross talk in the chamber. The same time window used to measure the efficiency was used to build the clusters (15 ns). The average cluster size has a very stable behaviour as function of the voltage and rate. For voltages in the range within which the chamber usually operates, the maximum cluster size is 1.2 strips, which indicates very small 
cross-talk.

The noise rate was estimated by subtracting the muon flux coming from the SPS beam dump $\left(\sim 30 \mathrm{~Hz} / \mathrm{cm}^{2}\right)$ from the rate measured with no source. This gives a noise rate of about $60 \mathrm{~Hz} / \mathrm{cm}^{2}$, which is rather high. The cosmic ray flux is negligible.

\section{Conclusions}

We have tested a four-gap RPC prototype in three facilities at CERN. It has been shown that the rate capability measurements at the PS are not reliable for high rates. From the measurements at the SPS, it was possible to see that an irradiation time longer than $\sim 1 \mathrm{~s}$ is needed for a stable operation of the chamber and that the variation of the rate within the burst must be taken into account when describing the efficiency drop. In GIF, the continuous irradiation allowed correct measurements of the rate capability to be made. In spite of the high resistivity of the melamine-phenolic plates of the prototype, the chamber reached $97 \%$ efficiency at $1.9 \mathrm{kHz} / \mathrm{cm}^{2}$. The time properties and cross-talk match the requirements for the LHCb muon system well.

\section{References}

[1] LHCb Technical Proposal, CERN/LHCC 98-4.

[2] R. Cardarelli et al., Nucl. Instr. and Meth. A 333 (1993) 39.

[3] M. Abbrescia et al., Nucl. Instr. and Meth. A 392 (1997)155-160

[4] P. Camarri et al., ATLAS Internal Note MUON-NO-226 (1998).

[5] F. Ceradini et al., Nucl. Instr. and Meth. A 340 (1994) 466-473

[6] E.Cerron-Zeballos et al., IV International Workshop on Resistive Plate Chambers and related Detectors, Naples, Italy, 15-16 October 1997.

[7] F. M. Newcomer et al., IEEE Trans. Nucl. Sci. 40(4) (1993) 630.

[8] M. Maggi et al., IV International Workshop on Resistive Plate Chambers and related Detectors, Naples, Italy, 15-16 October 1997.

[9] G. Carboni, talk at this workshop. 

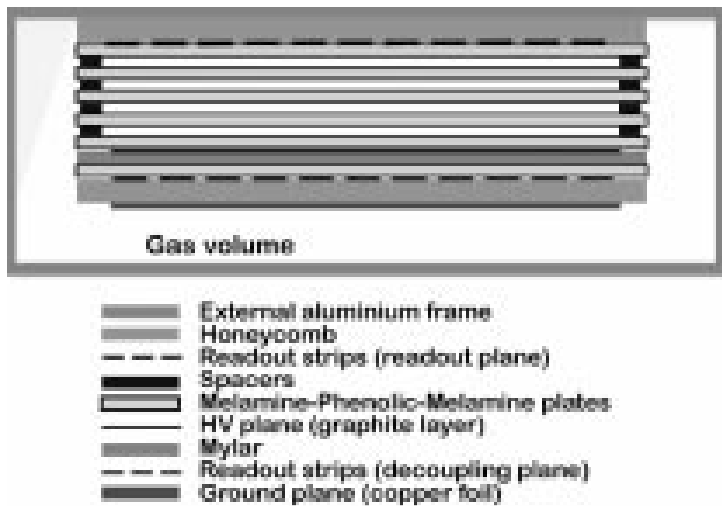

Fig. 1. Schematic of the resistive plate chamber. The HV plane is at the external face of the fifth melamine plate, from the top to the bottom part of the chamber. The honeycomb ensures the mechanical rigidity of the system.

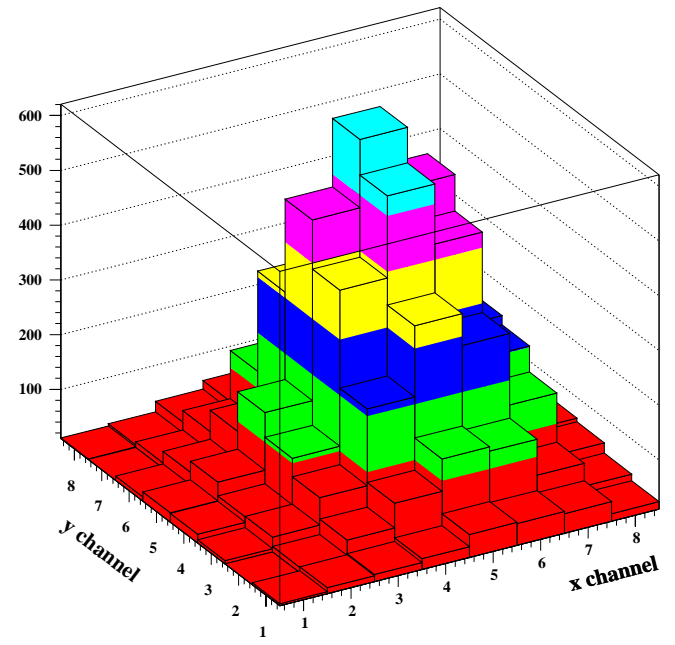

Fig. 2. Two-dimensional profile of the PS beam, determined by the hodoscope. 


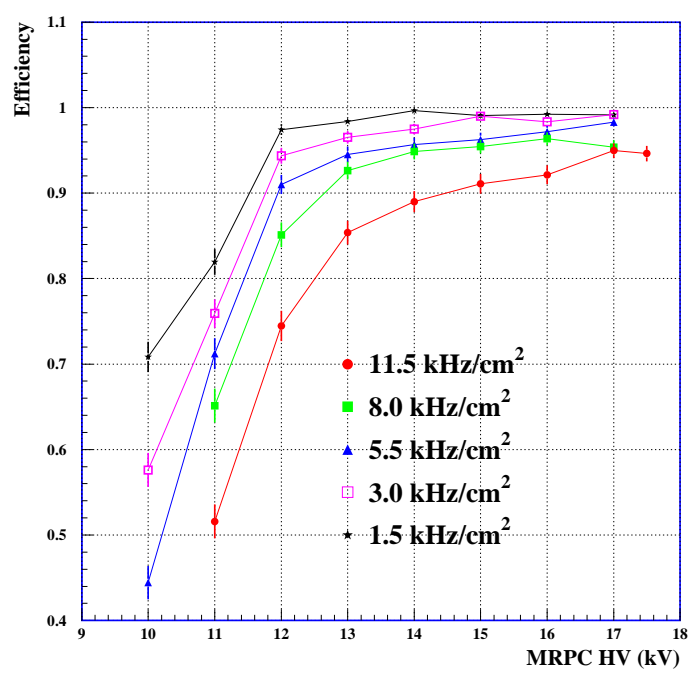

Fig. 3. Efficiency as a function of the high voltage, for several rates measured by the hodoscope in the PS.

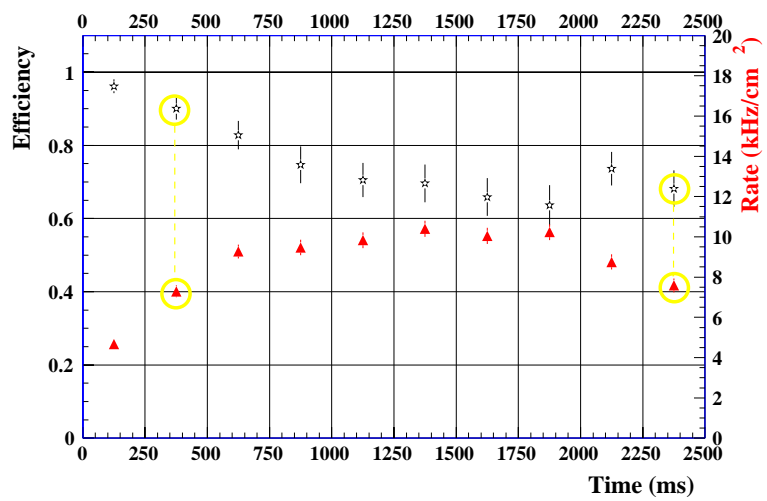

Fig. 4. Efficiency as a function of the time within the burst in the SPS hall (stars). The triangles are the rates for the same time intervals, measured at the hodoscope cell. 


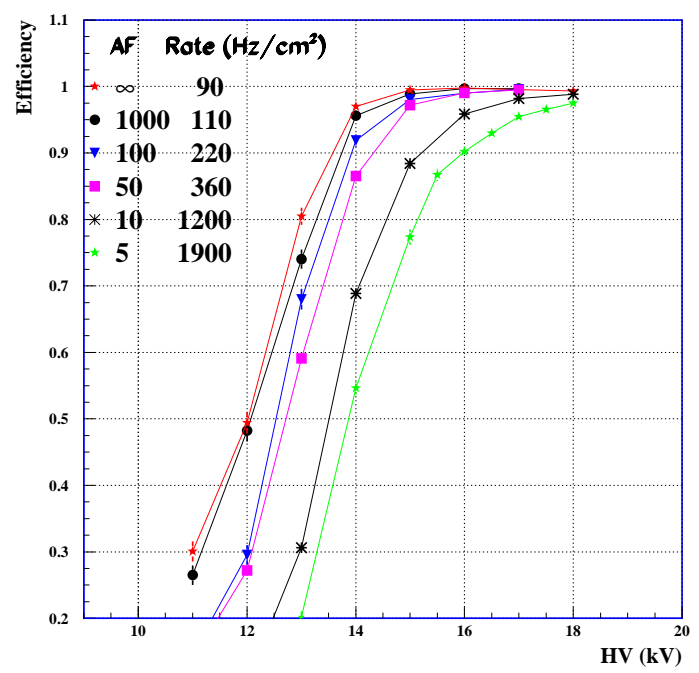

Fig. 5. Efficiency as a function of the effective rate of the MRPC, in the Gamma Irradiation Facility at CERN. The attenuation labelled " $\infty$ " refers to the data taken without source.

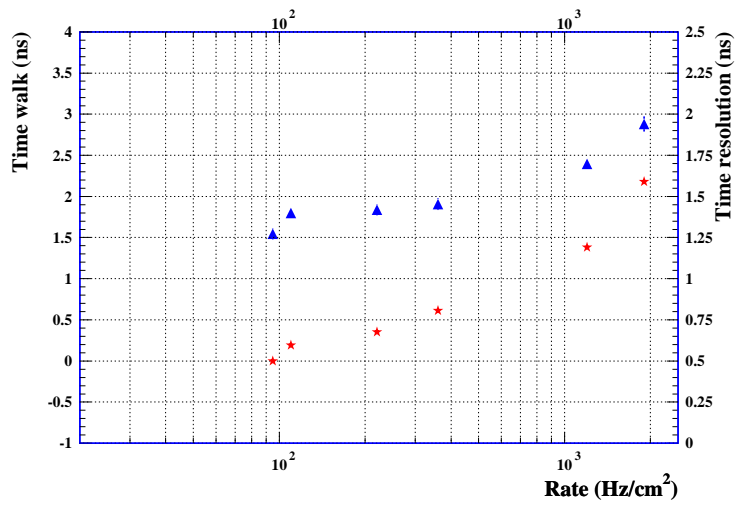

Fig. 6. Time walk (stars) and time resolution (triangles) as a function of the rate. 